\title{
P.Oxy. 2163 fr. 1: interpretazioni sceniche sul silenzio di Achille
}

Daniela Immacolata Cagnazzo (University of Bari Aldo Moro)

\section{P.Oxy. 2163 fr. 1: Scenic Interpretations of the Silence of Achilles}

\begin{abstract}
The earliest indirect evidence about silence on the Aeschylean stage is in Aristophanes, Frogs 911-913, where Euripides mentions an Achilles who is silent and fixed for a large part of a drama. The difficulty in interpreting these verses is due to the fragmentary trilogy of Achilles: in fact, only Mirmidones, thanks to P. Oxy. 2163, show some information about the use of silence in performance. In particular, fr. 11 of the papyrus attests a characterization of the veiled, seated, and twisted main character, just testified in the scholia vetera of Frogs. Thanks to this, it is possible to underline the silence typology, body language and performative elements of Achilles' character. Aeschylus creates an innovative theatre in which the absence of sound expresses feelings better than speeches do.
\end{abstract}

\section{Keywords}

silence; Aeschylus; fragmentary drama; aeschylean papyri; staging 
Il fr. 1 (= fr. 131 Radt) è uno degli undici frammenti raccolti attorno alla sigla di P.Oxy. $2163,{ }^{1}$ un papiro rinvenuto in Egitto a el-Bahnasa da Grenfell e Hunt agli inizi del Novecento, ma edito soltanto nel 1941 da Lobel all'interno del XVIII volume della collezione oxoniense dei papiri ossirinchiti. Dai Reports dei due studiosi non è possibile tracciare la storia del ritrovamento del frustolo e del suo arrivo sul continente europeo, ma ne è deducibile l'esatta località di provenienza grazie a un caso fortuito che ha consentito la ricostruzione testuale di una porzione di testo: infatti, il fr. 11 di P.Oxy. 2163 combacia con PSI XV 1472, recuperato da Breccia nello scavo del Kôm di Ali El Gammân nella campagna che portò alla luce altri frammenti eschilei.

Il papiro ha profonde lacerazioni e numerosi fori di diverse grandezze sparsi per tutto il kollema, mentre la scrittura è sbiadita in alcune parti (cfr. fr. 4 col. I ll. 2-3; fr. 8 ll. 2-4; fr. 11 1. 3), rendendo difficile l'interpretazione delle lettere e la lettura degli stichoi. I frammenti ${ }^{2}$ sono di diverse dimensioni, e fra questi le porzioni più ampie si riconoscono nei frr. 4 col. I, 8, 11, laddove le restanti riportano pochi moduli. I margini si sono conservati solamente nella parte superiore, e, benché non è dato stabilire con precisione quanto materiale sia andato perso, si noti che la lunghezza è di $2.8 \mathrm{~cm} \mathrm{ca} .^{3}$

La scrittura è una maiuscola rotonda, databile alla seconda metà del II secolo d.C., riconoscibile in quella dello scriba \#A3: ${ }^{4}$ presenta sinuosità del tracciato, rotondità delle forme, assenza di contrasto fra pieni e filetti, secondo la tipizzazione delle caratteristiche grafiche di uno stile estremamente regolare e calligrafico. Di interesse è l'uso di spiriti angolari, visibili al fr. 1: infatti, alla 1 . 2 è presente lo spirito anticipato sulla lettera O, mentre alla 1.4 è correttamente collocato sulla lettera $\Upsilon$; in quest'ultima linea compare una $\delta \iota \pi \lambda \tilde{\eta} \pi \varepsilon \rho \iota \varepsilon \sigma \tau \iota \gamma \mu \varepsilon ́ v \eta$, il cui senso resta oscuro. ${ }^{5}$

Ai fini della nostra indagine, relativa a come avvenisse la messinscena del silenzio del personaggio Achille, risultano di particolare interesse i versi del fr. 1 (= fr. 131 Radt), dal momento che documentano da un lato l'afasia di Achille, dall'altro l'effettiva presenza in scena del personaggio silente.

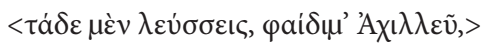

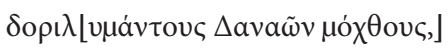

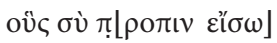

$\kappa \lambda ı \sigma i a\lfloor\varsigma$

oưvẹ [

1 Per una riproduzione digitale ad alta definizione si consulti il sito: POxy. Oxyrhyncus Online - Image Database.

2 Sono editi da Radt (1985) con la classificazione frr. 131 (= P.Oxy. fr. 1), **132a (= P.Oxy. frr. 2-9), 132b (= PSI $1472+$ P.Oxy. 2163 fr. 11).

3 Tale dato è confrontabile con le misure del margine superiore di PSI XI 1208, che è di $3.3 \mathrm{~cm}$ ca., e vale a comprovare che dimensioni così lunghe fossero proprie di un testo di manifattura pregevole; ciò trova riscontro anche nella larghezza piuttosto ampia $(2 \mathrm{~cm}$ ca.) dell'intercolumnio visibile fra la col. I e la col. II del fr. 4.

4 Cfr. Johnson (2004: p. 19).

5 Cfr. Lobel (1941: p. 26); McNamee (1992: pp. 33ss). 


\section{$\delta \eta \hat{i} .[$ \\ $\eta \rho .[$ \\ Toy[}

1 initium fabulae fuisse testantur Harp. $\pi$ 10, ¿vet. ad Ar. Ra. 992 [RVAM], Eusth. ad H. Od.

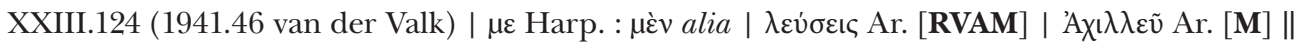

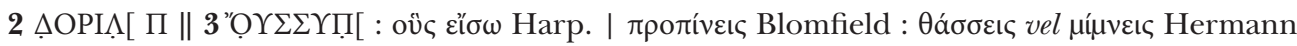

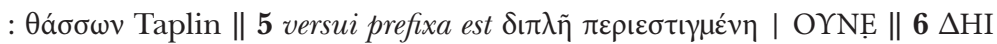

Queste cose tu vedi, o illustre Achille,

le pene dei Danai uccisi dalle lance,

che tu tradisci, standotene seduto

in una tenda $[\ldots]$

per questo

(tre versi con lemmi incerti)

L'attribuzione del frammento ai Mirmidoni $i^{6}$ è assicurata da alcune corrispondenze con la tradizione indiretta: si tratta del v. 1 presente negli scholia al v. 992 delle Rane di Aristofane (vet. 992a e rec. 992b Chantry, Tz. 992 Koster), e nel commento di Eustazio al v. 124 del XXIII canto dell'Odissea (1941.46 van der Valk). Inoltre, i primi quattro versi sono noti interamente da tradizione indiretta, grazie al Lessico di Arpocrazione ( $\pi$ 100, p. 222 Keaney).

La voce parlante è quella del Coro che cerca di convincere il figlio di Teti, seduto nella tenda (v. 4), a tornare sul campo di battaglia. È chiaro, dunque, che si è in un momento di crisi del combattimento, alimentato dall'assenza di Achille, il quale, lontano dalla guerra, si è rifugiato in un silenzio sdegnato e ostile a osservare il conflitto, mentre da tre giorni ${ }^{7}$ si trova nella propria tenda in totale immobilismo. Questo atteggiamento non verbale serve a delineare il carattere del personaggio e aiuta l'andamento drammaturgico, consentendo al Coro di annunciare nella parodo la preistoria della menis dell'eroe e le motivazioni che inducono il Pelide a chiudersi nel silenzio: è, infatti, l'affronto subìto da parte di Agamennone circa la schiava Briseide ad alimentarne l'astio verso i compagni, nonché la ferma volontà di non combattere.

L'apostrofe ad Achille, al v. 1 ( $\varphi \alpha^{\prime} \delta \mu^{\prime}$ 'Axı $\left.\lambda \lambda \varepsilon \tilde{v}\right)$, è pronunciato con toni in apparenza ossequiosi e affabili, e denuncia la presenza del personaggio sulla scena: egli, seppur silente, è visibile agli occhi del pubblico e, quasi sicuramente, resta lì immobile anche durante tutta la parte lirica cantata dal Coro. ${ }^{8}$

I coreuti raffigurano i membri di uno stesso gruppo molto vicino al protagonista, probabilmente uomini al suo comando, benché Achille sia un primus inter pares. Sull'identità del Coro una possibilità è che sia formato dalla guardia personale di Achille: più

6 Per una bibliografia accurata del dramma rinvio a Lucas De Dios (2008: pp. 425-447).

7 Cfr. Ar. Ra. 911-913.

8 Cfr. Taplin (1977: p. 112). 
genericamente, si può postulare ${ }^{9}$ che esso sia costituito da una parte dell'esercito dei Mirmidoni, ovvero sia da quegli «uomini scelti» di cui parla Nestore nell'Iliade (IX.165) e dai quali deriverebbe il titolo dell'opera.

I Mirmidoni erano un antico popolo della Tessaglia Ftiotide, estremamente fedele ad Achille, re di Ftia. Secondo una versione del mito traevano il nome dal re Mirmidone, figlio di Zeus e di Eurimedusa, sedotta dal padre degli dèi che aveva assunto l'aspetto di una formica; in un'altra versione, invece, essi discenderebbero dalle formiche, trasformate in uomini da Zeus, a cui Eaco, figlio della ninfa Egina e di Zeus stesso, si era rivolto per ripopolare l'isola di Egina: infatti, Era per vendicarsi del tradimento del coniuge aveva scagliato una pestilenza che aveva decimato la popolazione dell'isola. Essi costituiscono il nerbo delle forze militari guidate da Achille, e nell'Iliade sono descritti come guerrieri freddi e crudeli, con a capo cinque uomini: Alcimedonte (cfr. H. Il. XVI.197, XVII.467, 475, 481, 500), che guida il carro di Achille; Eudoro (cfr. H. Il. XVI.179, 186), figlio di Hermes; Fenice (cfr. H. Il. IX.223, 432, 690; XVI.196; XXIII.360), tutore di Achille; Menestio (cfr. H. Il. XVI.173), ucciso da Paride; Pisandro (cfr. H. Il. XVI.275), bravo nel lanciare il giavellotto. È, dunque, il racconto iliadico dei canti IX, XVI, XVII e XVIII che viene recuperato da Eschilo ${ }^{10}$ e che offre un parallelo con il plot del dramma, permettendone, se pur con le dovute cautele, una parziale ricostruzione, come vedremo in seguito.

La persona loquens pronuncia chiaramente e immediatamente il nome di Achille, per rendere nota al pubblico l'identità di quel personaggio fisso e silente sullo stage, che nei tratti e nell'atteggiamento doveva apparire differente dalla solita raffigurazione dell'eroe acheo, impavido e vigoroso. Interessante da segnalare è che "Achille» è un nomen loquens: composto da ä $\chi o \varsigma$ e $\lambda a o ́ \varsigma$, significa «dolore per il (suo) popolo», e, in effetti, l'eroe non fa che generare sofferenza fra la sua gente, anche se questo non significa che non se ne curi o che il dolore non si rifletta sulla sua persona. Achille è, dunque, una figura fortemente tragica: coinvolta involontariamente a combattere nella guerra di Troia, si trova ad essere influenzata dal volere degli dèi, in special modo dalla madre Teti, e a subire un oltraggio da Agamennone, finendo con il perdere il prezioso compagno Patroclo (cfr. H. Il. XVIII). Tuttavia, egli non è un personaggio statico, ma subisce un'evoluzione interiore: infatti, dalla fine del canto IX al canto XVI dell'Iliade termina l'offesa subita per il rapimento della schiava Briseide, e inizia una fase in cui esprime angoscia e ansia per Patroclo, che si mutano in dolore una volta saputo della sua morte.

L'evoluzione del carattere è possibile solamente attraverso il lutto, presente anche nella seconda sezione dei Mirmidoni: infatti, la perdita di Patroclo è il mezzo attraverso il quale si reintegra nel gruppo dei pares. Dal dolore nasce una nuova ira, la menis diventa

9 Hermann (1834: p. 140) sulla base dello scolio recenziore al v. 1264 delle Rane (cfr. $\Sigma$ rec. in Ar. Ra. 1264a Chantry) sostiene: «si chorus Myrmidonum et delectis ex Achillis exercitu viris constitit, qui cunctorum Myrmidonum nomine ad eum erant delegiti», quantunque Radt (1985: p. 240) replica: «in scholio illo non

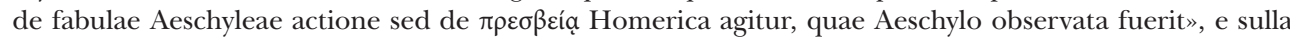
stessa linea si muoveva già Taplin (1972: pp. 69-70, n. 41).

10 Su Eschilo imitatore di Omero cfr. Moreau (1996: pp. 3-6). Per ulteriori indicazioni cfr. Kraias (2011) e Stama (2015) con relativa bibliografia. 
cholos, e si passa dalla dimensione pubblica a quella privata, il rifiuto iniziale del combattimento viene meno e il guerreggiare si trasforma in un atto di vendetta. «Il motivo conduttore della nuova vicenda è sempre l'ira di Achille, ma alla coppia Briseide/Achille si è sostituito il binomio Patroclo/Ettore»(Ciani 1988: p. 24). La $\varphi$ ı $\lambda$ ía verso Patroclo sovverte l'ordine dio-eroe: Achille non si lascia più guidare dalle decisioni di Zeus, ma agisce da uomo selvaggio, folle, accecato da un'ira ancora più violenta della precedente, che non si placa neppure con l'oltraggio del cadavere di Ettore (cfr. H. Il. XXII). Egli, dunque, non rispetta alcun codice eroico, morale o civile, ma si lascia trasportare da un'irrefrenabile ira, che si può arrestare solamente con l'intervento degli dèi (cfr. $\mathrm{H}$. Il. XXIV.113-115). Tuttavia, a partire dal canto XXIII con i giochi funebri in onore di Patroclo, Achille avvia il proprio percorso di reintegro nella comunità degli eroi, e la sua solitudine nel canto XXIV davanti a Priamo assume tratti differenti: egli è, qui, non il giovane ribelle, ma l'uomo consapevole di essere guerriero e sovrano di un popolo. Il ritorno all'equilibrio e all'ordine, presente nel testo iliadico è recuperato da Eschilo nei Frigi ovvero il Riscatto di Ettore, ove era rappresentata, a conclusione della trilogia su Achille, la magnanimità dell'eroe verso il nemico. ${ }^{11}$

Di Benedetto (1967) ha posto in evidenza come i vv. 1-2 del fr. 132 Radt (= Ar. Ra. 1264-1265) sarebbero un'eco dei vv. 1-2 del nostro frammento, in quanto vi sarebbe

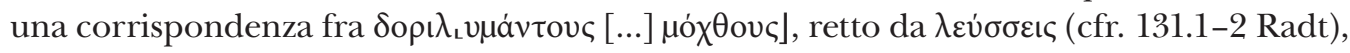

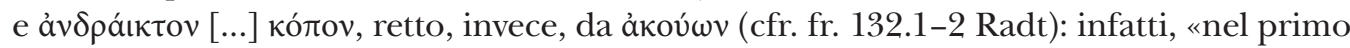
caso la situazione di disagio degli Achei viene presentata semplicemente come evidente ad Achille (e agli spettatori); nel secondo caso invece si presuppone quasi certamente un racconto che qualcuno deve aver fatto delle difficoltà in cui l'esercito greco si trova». Pertanto, stando sempre a Di Benedetto, nel fr. 132 Radt vi è sì una ripresa dell'argomento da parte della persona loquens, che così si inserisce nella stessa scena del fr. 131 Radt, ma a parlare non è il Coro, bensì un altro personaggio. ${ }^{12}$

Achille, nonostante i diversi appelli rivoltigli direttamente, resta in silenzio. Il rifiuto della parola serve ad accrescere la spiccata individualità del protagonista, che è raffigurato come spietato e slegato dai vincoli familiari o etico-politici. L'eroe appare lontano dai valori della đódıৎ dell'Atene del V secolo a.C., ove non si dà spazio al singolo, ma alla collettività, e in cui il desiderio del bene comune è più forte dell'amor proprio. Il suo essere aristocraticamente solitario si riflette nella modalità con cui gli Achei intendono punire il suo tradimento: il linciaggio, a cui l'eroe stesso si riferisce (cfr. fr. 132c.1 Radt), è la sentenza inflitta per eccellenza dalla giustizia popolare. ${ }^{13}$

11 Cfr. Hadjicosti (2007: p. 150).

12 In base a ciò, Di Benedetto (1967) ha postulato che vi fosse una scena con Taltibio, subito dopo la parodo e prima dell'arrivo di Fenice. La supposizione non è da escludere, in quanto, contrariamente alla Niobe, non si posseggono per i Mirmidoni informazioni più o meno dettagliate circa la durata del silenzio del protagonista, sebbene i riferimenti in nostro possesso facciano propendere per un intervento di Achille in un fase non troppo avanzata del dramma, dal momento che egli interagiva non poco con gli altri attori (cfr. **132b, 132c, 134, 135 Radt).

13 Sul linciaggio come sentenza storica vd. Edmunds (1996: pp. 90-91). 


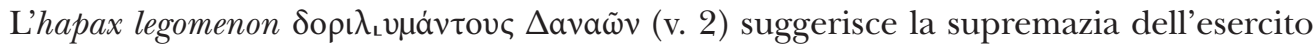
troiano su quello acheo, il primo sempre più feroce, il secondo incapace di resistere alle armi nemiche a causa dell'assenza di Achille dal campo di battaglia. Anche nel fr.

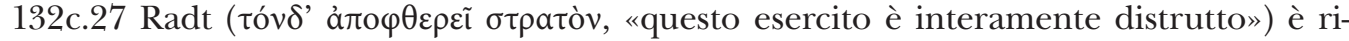
badito il momento di difficoltà che l'esercito acheo si trova a dover affrontare, vivendo

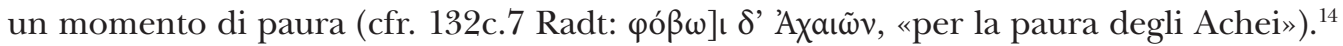

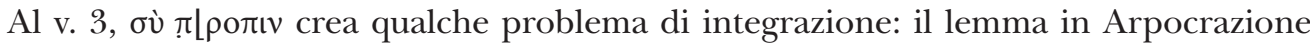

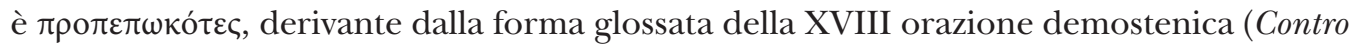
Ctesifonte), e, secondo Taplin (1972: p. 66, n. 27), non vi è ragione per asserire che il vocabolo fosse usato da Eschilo nella stessa diatesi e forma. Il où, leggibile nel frammento

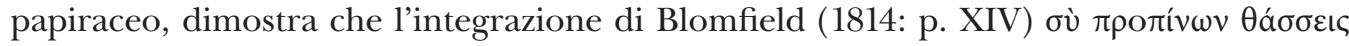
sia attendibile, ma anche altre soluzioni potrebbero essere valutate, ${ }^{15}$ fra cui ov̀ $\pi \rho$ o $\operatorname{civ\varepsilon ı\varsigma }$ Өá $\sigma \omega v$, proposta da Taplin (1972: p. 66), sebbene non vi siano argomenti sufficienti per accertare che il verbo corretto sia $\theta$ á $\sigma \omega$.

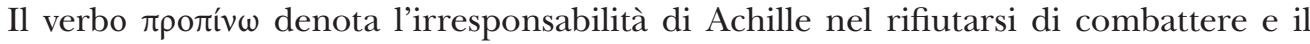
tradimento della fiducia riposta in lui da parte dell'intero campo acheo, che ne riconosce il valore supremo. Si tratta, dunque, di un uso metaforico del lemma, desunto dal contesto simposiale, in cui «qui socios prodebant, et provocati non respondebant,

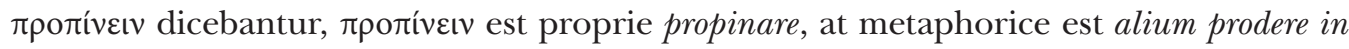

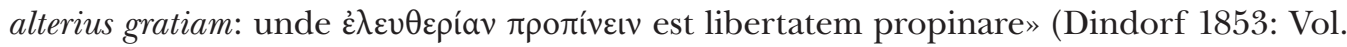
II., p. 398). L'ambiguità metaforica, definita paradossale da Moreau (1996: p. 13), è voluta: il verbo contiene in sé il liguaggio «de la fête bacchique et de l'amour», che, come la guerra, sono tutti e tre contrassegnati da legami, quali rispettivamente il rito sacrificale, l'affetto, l'alleanza militare. Difatti, bere nella stessa coppa è un rituale simbolico, con cui si esprime un atto di fiducia e condivisione, è essere un pari e lottare per gli stessi valori, è dichiararsi compagno e stringere un patto d'alleanza. Tuttavia, se queste caratteristiche vengono meno, il tutto si rompe, e colui che ha violato il patto di fedeltà diviene un traditore. Al Pelide, dunque, non resta che bere le sofferenze dei Danai, il loro sangue; ne consegue, alla luce di $\pi \rho \pi_{i} v \omega$, che l'appellativo «illustre, glorioso», usato nel verso incipitario, assume nuovi contorni ironici, che il pubblico non avrebbe potuto cogliere da subito.

Il riferimento al tradimento di Achille diviene un motivo costante nel dramma, almeno da quel che si è conservato: è, infatti, attestato anche nel fr. 132a.8.5 ( $\mu \grave{\eta} \pi \rho \circ \delta \tilde{\omega} \varsigma$ ) e nel

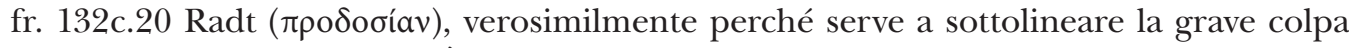
di cui l'eroe si è macchiato. È che nel resto dei versi, a noi ignoti, venissero descritte le difficoltà che dovevano affrontare gli Achei senza l'intervento decisivo del figlio di Teti, nonché i voleri dei capi della spedizione che si erano incontrati poco prima nella tenda di Agamennone, così come viene narrato da Omero (cfr. H. Il. IX.89-90). È in questo frangente che viene proposto da Nestore, il più saggio fra gli eroi achei, di compiere un'ambasceria per riempire di doni e convincere con parole suadenti Achille, restio a

14 Sulla paura degli Achei come paura di Achille vd. Michelakis (2002: p. 27).

15 Cfr. Dindorf (1853: Vol. I, p. 260). 
tornare in battaglia (cfr. H. Il. IX.92-113). Sono persuasa dell'idea che anche nei Mirmidoni avvenisse inizialmente un simile approccio adulatorio, e che soltanto in un momento successivo si decidesse di ricorrere a un'azione violenta, per cui era decretato dal consiglio dei capi dell'esercito che Achille venisse punito con la lapidazione, in quanto non demordeva nella decisione di non combattere e non parlare, macchiandosi di tracotanza, colpa riconducibile a un carattere tirannico e, dunque, inammissibile per gli altri eroi. Similmente, Barabino (1956: p. 60) postula una scena intermedia (fra Achille e Antiloco), che segue l'ambasceria, in quanto la minaccia di lapidazione «non poteva essergli rivolta dal personaggio dell'ambasceria, che gli offriva dignitose proposte», come avviene in H. $I$. IX.260-276, in cui Odisseo prospetta i doni che Agamennone gli concederà, qualora ritorni a guerreggiare.

La resa italiana del termine greco $\kappa \lambda \iota \sigma i \alpha_{\llcorner} \varsigma$ (v. 4) non consente in modo adeguato di comprendere le caratteristiche di una simile struttura, di cui già si faceva cenno nell' $I$ liade, in particolare nel canto XXIV ai vv. 448-456, ove è descritta dettagliatamente per evidenziare la grandezza di Achille, che è sì un soldato, ma anche un sovrano.

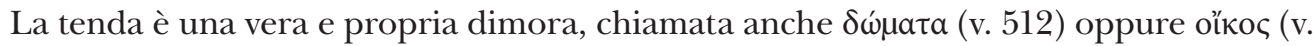

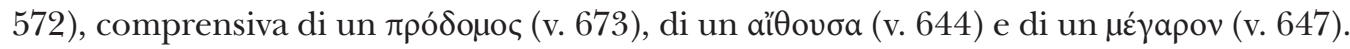

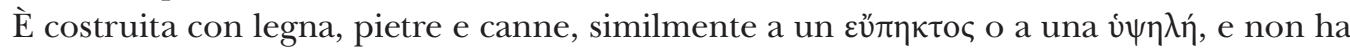
nulla di effimero né di temporaneo, ma enuncia la personalità di un personaggio che è abituato più al lusso dei palazzi che alla frugalità degli accampamenti militari. Sembra inverosimile che in scena fosse collocata la struttura lignea narrata da Omero, ma vi era presumibilmente qualcosa che potesse dare l'idea agli spettatori di una simile ambientazione, tale che potesse anche suggerire l'idea dell'ambiente interno. Del resto, l'azione degli attori e del Coro era limitata: Achille era costantemente seduto in scena, la fisicità e gestualità dichiaravano apertamente quel che non diceva con le parole, i personaggi dovevano relazionarsi col personaggio silente, adeguandosi alla sua immobilità.

Sulla base della tradizione diretta e indiretta si possono dedurre alcuni dettagli che consentono, se pur con profonde incertezze, di ricostruire la trama dei Mirmidoni, la quale doveva incominciare in medias res, come si rileva dal fr. 131 Radt, e al Coro era affidato il compito di introdurre gli spettatori agli antefatti della vicenda, narrando il motivo per cui Achille si era allontanato dal campo di battaglia, rifugiandosi nella propria tenda, e fornendo una spiegazione sul perché dell'ambasceria che li aveva condotti lì. Alla parodo faceva seguito l'arrivo dell'ambasceria, che dava così inizio alla prima parte del dramma, in cui si concentrava l'ostinazione di Achille, il silenzio irato e gli interventi dei messaggeri. Dopo il ritorno di Achille alla parola (cfr. frr. **132b-**132c Radt) grazie al discorso di Fenice, vi era la descrizione dell'incendio delle navi (cfr. frr. 133-134 Radt), che probabilmente lo convinceva a inviare Patroclo e altri uomini a combattere in sua vece. A questo punto, si apriva la seconda parte del dramma, che dava avvio a una nuova sezione, i cui temi dominanti erano l'amore e la morte per il compagno Patroclo (cfr. frr. 134a-139 Radt). ${ }^{16}$ Il dramma si chiudeva forse con un threnos per Patroclo o con

16 In merito al rapporto fra Achille e Patroclo nei Mirmidoni vd. Garzya (1995: pp. 53-56); Moreau (1996: pp. 16-20); Michelakis (2002: pp. 41-53); Hadjicosti (2007: pp. 127-136). 
l'apparizione di Teti che prometteva le nuove armi al figlio (a questo dialogo si riferirebbe forse il fr. 140 Radt).

Per quanto concerne l'ambasceria, Sommerstein (2008: pp. 339, 342) è dell'idea che non fosse prevista nella trama, perché sarebbe stata difficile da rendere sul piano performativo vista la presenza di due soli attori. Tuttavia, questo significherebbe ignorare

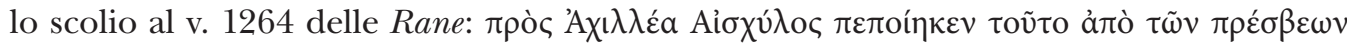
(«Eschilo ha composto questo dall'ambasceria per Achille»); dove l'attenzione è puntata proprio sull'ambasceria, in cui sono coinvolti più personaggi, visto l'uso del plurale $\pi \rho \varepsilon \varepsilon \sigma \beta \varepsilon \omega v$. Inoltre, non si spiegherebbero le parole di Achille al v. 7 del fr. **132b Radt $(\pi \circ \lambda \lambda \tilde{\omega} v$ ảkov́ $\omega v \delta v \sigma \tau o ́ \mu \omega v)$, le quali alludono a più di un intervento per persuaderlo a tornare al combattimento. È, dunque, difficile appoggiare l'idea di Sommerstein, e va, invece, confermata la scena di presbeía analoga a quella iliadica. ${ }^{17}$

Chi erano i componenti dell'ambasceria nei Mirmidoni? Qualche idea può essere desunta da un confronto con il IX canto dell'Iliade, in cui la proposta di condurre degli uomini da Achille viene fatta da Nestore ad Agamennone (cfr. vv. 168-170), ${ }^{18}$ al fine di arginare i pericoli che gli Achei si trovano ad affrontare; vengono così inviati Fenice, Odisseo, Aiace e due araldi. Il primo a prendere parola è Odisseo (cfr. vv. 201-306), seguito da Fenice (cfr. vv. 434-605), e, infine da Aiace (vv. 624-642). Tuttavia, se qui Achille prende una decisione già dopo il discorso di Odisseo, nei Mirmidoni preferisce essere silente, come a voler tardare la risposta da riferire ad Agamennone. Inoltre, nell'opera eschilea si presume che gli eroi parlassero in un ordine diverso dal canto omerico, se Fenice è l'unico a far riacquistare la parola ad Achille, dopo che questi ha dovuto udire «molti discorsi» (cfr. fr. **132b Radt). Malgrado le somiglianze tematiche, gli unici dati certi che emergono dai frammenti sono la presenza di Fenice (cfr. **132b Radt) e di Antiloco (cfr. fr. 138 Radt), mentre resta incerta la presenza di Odisseo, a cui sarebbe ricondotta la figura del parlante nel fr. **132a fr. 8.4 Radt.

Sul piano performativo si rileva che il luogo dell'azione è la tenda di Achille (fr.

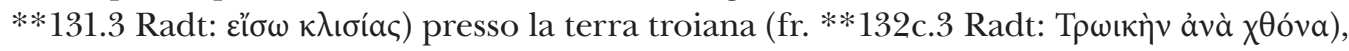

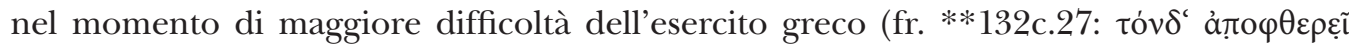

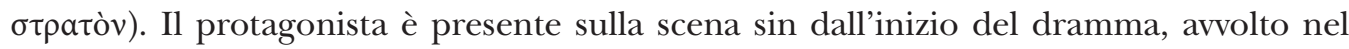
proprio mantello, seduto su una sedia o uno sgabello, sicuramente in solitudine, laddove nell'Iliade (IX.186-191) si racconta che suona la cetra, ascoltato da un Patroclo silenzioso. L'essere seduto e coperto dal manto è una volontaria interruzione del contatto comunicativo, sicché una ripresa della comunicazione equivale a una diversa esecuzione di quest'ordine che sollecita il ripristino delle condizioni scenico-dialogiche normali, per cui bisogna immaginare che Achille svelasse il proprio viso una volta che interloquiva con Fenice e gli altri attori. Pertanto, si è davanti a un uso del silenzio che è sia performativo sia funzionale all'espressione del carattere del personaggio. L'afasia, infatti, diventa simbolo dell'ira che lo possiede violentemente e lo chiude nei propri pensieri.

17 La pittura vascolare documenta in alcuni vasi attici di fine VI e inizi V secolo a. C. la presenza dell'ambasceria iliadica; su ciò cfr. Johansen (1967).

18 Sull'ambasceria ad Achille dell'Iliade cfr. Griffin (1995: pp. 51-53). 
La presenza in scena della tenda, al cui interno (cfr. v. 3: عl $\sigma \omega)$ si troverebbe Achille seduto, velato e silente (cfr. Ar. Ra. 911-913), implica problemi esegetici e scenico-testuali di non facile risoluzione: infatti, in che modo era rappresentata la tenda? Innanzitutto, va precisato che non ci sono dati materiali su cui basare con certezza la ricostruzione scenica né tantomeno scenografica; in secondo luogo, la mancanza di una cronologia sulla rappresentazione del dramma amplia il margine di incertezza, poiché le notizie sul modo di mettere in scena le opere teatrali di inizio $\mathrm{V}$ secolo a.C. sono pressoché nulle;

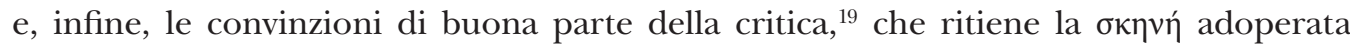
dall'Orestea in poi (laddove, per il periodo precedente, si considera l'intera orchestra come

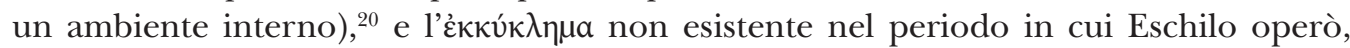
condizionano le ipotesi di messinscena. Se ci si attiene a quest'ultimo punto, bisogna

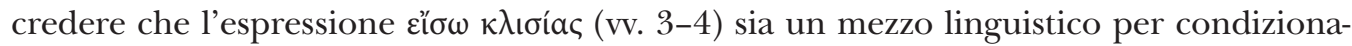
re l'immaginazione dello spettatore e catapultarlo volutamente nell'ambiente designato dal tragediografo, cioè la tenda d'Achille, altrimenti non rappresentata. È, dunque, un forzare la mente di chi osserva per ricreare uno scenario illusorio grazie all'ausilio della parola; pratica non estranea ad un teatro che non conosceva i cambi frequenti di scenografia. In questo modo, sia il Coro sia l'attore che impersona Achille si troverebbero in uno spazio aperto, con qualche oggetto di scena indicante l'ambiente interno della

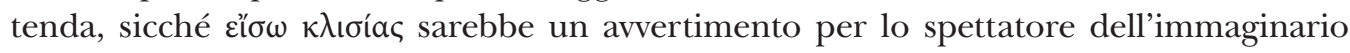
ingresso del Coro nella tenda. La soluzione scenica prospettata da Taplin (1972: p. 69) potrebbe essere condivisibile, lo è meno quella di Croiset (1894: p. 153), per cui Achille è seduto dinnanzi all’ingresso della tenda. Döhle (1967: p. 118), invece, sul confronto con l'iconografia di vasi attici del V secolo a. C., argomenta la congettura di un telone istoriato che consente all'azione di svolgersi tutta all'interno della tenda; ed ancora,

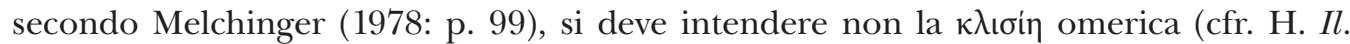
IX.185), costruita in legno, ma una struttura piů semplice, magari mobile, il cui interno

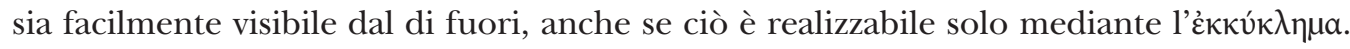

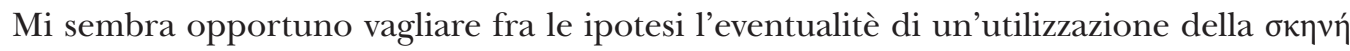
strutturata con una porta, indicante l'ingresso, e un tetto, per dare l'idea di essere nell'ambiente interno e chiuso della tenda del protagonista.

In definitiva, grazie alla testimonianza del fr. 1 di P.Oxy. 2163 che supporta la testimonianza di Ar. Ra. 911-913, si può affermare che il silenzio di Achille nei Mirmidoni è radicato nella sua essenza di eroe colpito nella $\tau \mu$,́, per cui è, dapprima, sopraffatto dagli eventi (cfr. frr. 134, 138 Radt), poiché spinto dall'ira e dall'orgoglio, e, in seguito, rimpiange la scelta di aver mandato a combattere al suo posto Patroclo e gli altri compagni. Da qui, si dà avvio a un nuovo argomento, che verrà sviluppato nel dramma successivo dell'Achilleide, cioè i Frigi ovvero il Riscatto di Ettore, dove il silenzio (in un'accezione differente) avrà, ancora una volta, una funzione chiave per la messinscena, poiché Achille, sopraffatto dal dolore per la perdita dell'amasio morto in battaglia per uno scambio

19 Cfr. Taplin (1972: p. 67, n. 31).

20 «That is to say that the audience would imagine, if asked, that the scene being played before them in the open air was in fact set indoors» (Taplin 1972: p. 67). 
con la sua persona, preferirà soffrire nella più profonda reticenza: ${ }^{21}$ il dolore, infatti, sarà ineffabile a tal punto da rendere le parole insufficienti per esprimere un lutto così desolante.

\section{Bibliografia}

Barabino, G. (1956). I 'Myrmidones' di Accio. In Antidôron Hugoni Henrico Paoli oblatum. Miscellanea Philologica (pp. 57-72). Genova: Ist. di Filologia Classica.

Bartoletti, V. (1966). Un frammento dei 'Myrmidones' di Eschilo. In A. E. Samuel (Ed.), Essays in Honor of C. Bradford Welles (pp. 121-123). New Haven: American Society of Papyrologists.

Blomfield, C. J. (1814). Aeschyli Persae. Cambridge: University Press.

Ciani, M. G. (1988). Omero. L'ira di Achille (Iliade I). Venezia: Marsilio.

Cordano, F. (1998). Achille desiste dall'ira. In M. Sordi (Ed.), Responsabilità, perdono e vendetta nel mondo antico (pp. 3-8). Milano: Vita e Pensiero.

Croiset, M. (1894). Eschile imitateur d'Homère dans les Myrmidons, les Néréides et les Phrygiens. Revue des Études Grecques, 7, 151-180.

Di Benedetto, V. (1967). Il silenzio di Achille nei 'Mirmidoni'di Eschilo. Maia, 19, 373-386.

Dindorf, G. (Ed.). (1853). Harpocrationis Lexicon in decem oratores atticos. Groningen: Bouma's boekhuis.

Döhle, B. (1967). Die 'Achilleis' des Aischylos in ihrer Auswirkung auf die attische Vasenmalerei des 5. Jahrhunderts. Klio, 49, 63-149.

Edmunds, L. (1996). Theatrical Space and Historical Place in Sophocles' Oedipus at Colonus. Lanham - Boulder - New York - London: Rowman \& Littlefield.

Garzya, A. (1995). Sui frammenti dei 'Mirmidoni’ di Eschilo. In J. A. López Férez (Ed.), De Homero a Libanio. Estudios actuales sobre textos griegos (Vol. 2 ; pp. 41-56). Madrid: Ediciones Clásicas.

Griffin, J. (Ed.). (1995). Homer: Iliad. Book Nine. Oxford: University Press.

Hadjicosti, I. (2007). Aischylos and the Trojan Cycle. The Lost Tragedies (Diss. thesis). London: University of London.

Hermann, G. (1834). De Aeschyli Myrmidonibus, Nereidibus, Phrygibus (Opuscula, V; pp. 136-163; Diss. thesis). Leipzig: Staritz.

Johansen, K. F. (1967). The Iliad in Early Greek Art. Copenhagen: Munksgaard.

Johnson, W. A. (2004). Bookrolls and Scribes in Oxyrhynchus. Toronto: University Press.

Kraias, G. (2011). Epische Szenen in tragischem Kontext: Untersuchung zu den Homer-Bezügen bei Aischylos (Prismata). Bern: Peter Lang.

Lobel, E. (1941). P.Oxy. 2163. In E. Lobel, C. H. Roberts, \& E. P. Wegener (Eds.), The Oxyrhynchus Papyri, Part XVIII. London: Egypt Exploration Society.

Lucas De Dios, J. M. (2008). Esquilo. Fragmentos - Testimonios. Introducciones, traducciòn y notas. Madrid: Editorial Gredos.

McNamee, K. (1992). Sigla and Select Marginalia in Greek Literary Papyri. Bruxelles: Fondation Égyptologique Reine Élisabeth.

21 Cfr. T 1.6 Radt. 
Melchinger, S. (1978). Das Theater der Tragödie. Aischylos, Sophokles, Euripides auf der Bühne ihrer Zeit. München: Beck.

Michelakis, P. (2002). Achilles in Greek Tragedy. Oxford: University Press.

Moreau, A. (1996). Eschyle et les tranches des repas d'Homère: La Trilogie d'Achille'. In A. Moreau (Ed.), Panorama du théâtre antique: D'Eschyle aux dramaturges d'Amérique Latine (Cahiers du GITA, 9; pp. 3-29). Montpellier: Groupe Interdisciplinaire du Théâtre.

POxy. Oxyrhynchus Online - Image Database. The Oxyrhynchus Papyri. University of Oxford. [Retrieved 19.04.2019; http://163.1.169.40/cgi-bin/library? e=q-000-00-0POxy-00-0-0-0prompt10--4--0-11-1-en-50-20-about-2163-00031-001-0-0utfZz-8-00\&a=d\&c=POxy\&cl=search\&d=HASH0101d03f21391ec2a7a74ad3.oxy].

Radt, S. L. (Ed.). (1985). Tragicorum Graecorum Fragmenta (TrGF), III: Aeschylus. Göttingen: Vandenhoeck \& Ruprecht.

Sommerstein, A. H. (2008). Aeschylus, III: Fragments. Cambridge - London: Harvard University Press.

Stama, F. (2015). Il riscatto del corpo di Ettore: una rivisitazione mercantesca in Eschilo. Lexis, 33, 71-79.

Taplin, O. (1972). Aeschylean Silences and Silences in Aeschylus. Harvard Studies in Classical Philology, 76, 57-97.

Taplin, O. (1977). The Stagecraft of Aeschylus. Oxford: University Press.

Daniela Immacolata Cagnazzo / daniela.cagnazzo@uniba.it

Department of Humanities (DISUM)

University of Bari Aldo Moro

Palazzo Ateneo - Piano 2 , Piazza Umberto I, 1, Cap 70121 Bari, Italy

This work can be used in accordance with the Creative Commons BY-SA 4.0 International license terms and conditions (https://creativecommons.org/licenses/by-sa/4.0/legalcode). This does not apply to works or elements (such as image or photographs) that are used in the work under a contractual license or exception or limitation to relevant rights. 
